\title{
EDITORIAL
}

\section{Very little is new in nanoscience}

In conceiving a special section for European Journal of Physics, a number of selection criteria will usually operate. Primarily, the chosen theme needs to be a subject area that is sufficiently broad to offer a range of opportunities for pedagogic exploration. Although the teaching of science must always lag a little behind forefront research, many of the most exciting developments occur just as the wisdom in a new subject area is consolidated and systematized. At such a juncture, as new topics are being added to the curriculum, there is fresh scope to reflect on how best to approach the art of instruction, and to consider what principles might be conveyed in any new course of lectures. The result is often a double bonus; the pleasure of introducing new material reflecting current developments and imminent technologies is compounded by the discovery of new contexts for the delivery and grounding of fundamental concepts.

The compilation of papers that follows, focusing on nanoscience and technology, amply illustrates these principles. Here is a topical area within which much is decidedly new, reflecting technical possibilities that have in the past been impossible to achieve. Techniques such as dip-pen nanolithography [1] and atomic/molecular force microscopy [2], which have provided us with the technical capability of manipulating matter at nanoscale dimensions, are already being supplemented by newer optical methods [3]. It is nonetheless salutary to reflect that the actual existence of nanoparticles is nothing new. Buckminsterfullerene, that most iconic molecule heralding the arrival of nanoscience, has proved to be present in a wide range of natural materials including different types of soot [4]. Metallic nanoparticles are also now known to be responsible for a curious red hue, exhibited by a famous ancient Roman glass vase on its illumination from within [5].

The recent surge of interest in nanoscientific topics has come to pass not only because of the realization of nanomanipulation technologies. Nor, despite the folklore, can its provenance be traced to speculations by Feynman [6]. Of course, there isn't any new kind of physics that specially operates at the nanoscale, but access to matter on that scale now affords unprecedented opportunities to thoroughly exploit a variety of exotic phenomena such as quantum and nanophotonic effects. Surface phenomena also gain a greatly increased significance [7]. Even the well-known link between reactivity and surface-to-volume ratio becomes a grand principle, a major determinant of physical properties, when it operates over nanoscale dimensions. It is no secret that some relatively new materials and treatments-such as the titanium dioxide coatings on self-cleaning windows, or anti-bacterial coatings of silver in refrigerators-become most efficacious when their particle sizes become vanishingly small. Almost nothing is new in nanoscience.

In the pages that follow there is much to inform and inspire others with the singular educational opportunities afforded by a subject that has rather quickly come into vogue. Some of the material, such as the description of photonic crystals, provides an exemplary basis to reinforce and develop concepts that students will already be familiar with, extending their comprehension whilst also introducing them to an area in which further technical developments are taking place. Other papers, such as the contribution on quantum coherence, concerning phenomena that are uniquely nanoscale in origin, might be used to introduce more advanced, ground-breaking material. Here, the major applications may still be some way off in the future.

Although a great deal of media hype has surrounded the general public's dawning perception of nanotechnology, it should not be forgotten that there are indeed many serious issues to consider, as products of the technology are rolled out into society [8]. The early 
recognition of such a dimension in a comparatively young science can only be applauded. Nanoscience affords a particularly fertile ground for cultivating student engagement with the broader societal implications of their studies. It embraces topics that invite reflection and debate-and where such debate moves into the public arena, it very much needs participation by properly informed individuals. Here is an opportunity not only to impress upon the next generation of scientists the need to be involved in public awareness, but also to equip them with a sound understanding of the scientific principles involved in nanotechnology.

It is a great pleasure to have found, amongst the rapidly escalating number who work in this area, highly esteemed authors willing to produce papers especially for this special section of European Journal of Physics. My thanks are due to them for delivering almost on time, which is as much as one can reasonably ask. I trust they and many other readers will find the result of their endeavours thoroughly worthwhile.

\section{References}

[1] Piner R D, Zhu J, Xu F, Hong S and Mirkin C A 1999 Science 283 661-3

[2] Noy A (ed) 2008 Handbook of Molecular Spectroscopy (New York: Springer)

[3] Dienerowitz M, Mazilu M and Dholakia K 2008 J. Nanophoton. 2021875

[4] Kroto H 1988 Science 242 1139-45

[5] Freestone I, Meeks N, Sax M and Higgett C 2007 Gold Bull. 40 270-7

[6] Ball P 2009 Chem. World 6 58-62

[7] Andrews D L and Gaburro Z (ed) 2007 Frontiers in Surface Nanophotonics (New York: Springer)

[8] Munshi D, Kurian P, Bartlett R V and Lakhtakia A 2007 Futures 39 432-52

\section{David L Andrews}

University of East Anglia, UK

\section{Guest Editor}

